\title{
Notch4, uncovering an immunomodulator in allergic asthma
}

\author{
Beatriz Moya ${ }^{1}$, Manali Mukherjee ${ }^{2}$, and Parameswaran Nair ${ }^{2}$ \\ ${ }^{1}$ Hospital Universitario 12 de Octubre \\ ${ }^{2}$ McMaster University
}

May 27, 2021

Title: Notch4, uncovering an immunomodulator in allergic asthma

Authors: Beatriz Moya ${ }^{\mathrm{a}}$, Manali Mukherjee ${ }^{\mathrm{b}}$ and Parameswaran Nair ${ }^{\mathrm{b}}$

a. Department of Allergy, Hospital Universitario 12 de Octubre, Madrid, Spain

b. Division of Respirology, Department of Medicine, McMaster University, Hamilton \& Firestone Institute for Respiratory Health, St Joseph's Healthcare, Hamilton, ON, Canada

Correspondence to : Beatriz Moya. Allergy Department. Hospital Universitario 12 de Octubre, Madrid, Spain

Email:drbeatrizmoya@gmail.com

Acknowledgements : Dr. Mukherjee is supported by investigator award from Canadian Institutes of Health Research and Canadian Allergy, Asthma, and Immunology Foundation. She has received honorarium from AZ, GSK and her university has received grants from Methapharm Speciality Pharmaceuticals. Dr. Nair is supported by the Frederick E. Hargreave Teva Innovation Chair in Airway Diseases. He has received honoraria from AZ, Sanofi, Teva, Merck, Novartis and Equillium and his university has received research grants from AZ, Teva, Sanofi, Novartis, BI and Methapharm. The authors recognize Dr. Anna Globinska for graphical abstract design and Dr. Rodrigo Jiménez-Saiz for critical review of the manuscript.

Keywords: Allergic asthma; Airway inflammation; Th2 cell; Th17 cell; Treg cell; Notch4 receptor

Abbreviations: Th, T helper; UFPs, pollutant ultrafine particles; AMs, alveolar macrophages; Treg cells, regulatory T cells; ILC2s, type 2 innate lymphoid cells; GDF15, cytokine growth and differentiation factor 15; IL, interleukin; IL-6R, interleukin-6 receptor; IL-4R, interleukin-4 receptor

Word count: $918 / 1000$

\section{Main text:}

Asthma is a chronic inflammatory disease of the airways that causes bronchial hyperreactivity, mucus overproduction, airway remodelling and narrowing. Allergic asthmatic patients generally present a predisposition toward $\mathrm{T}$ helper $(\mathrm{Th}) 2$ inflammation that is maintained by allergen-driven innate factors and downstream activation of Th2 cells. In this regard, Notch signalling activates the key Th2 transcription factor Gata-3. In fact, Notch is considered as a master regulator of Th cells to terminally differentiate into Th1, Th2, Th17 lineages by directly inducing the respective transcription factors and cytokines. ${ }^{1}$ The latest findings by Harb et al.,further report how Notch signalling enables an inflammatory response in the allergic airways by altering T-regulatory (Treg) cells into effector Th2 and Th17 cells. ${ }^{2}$

The Notch family is formed by 4 transmembrane protein receptors (Notch1-4) which regulate different cell signalling pathways. ${ }^{1}$ Notch4 is involved in a number of cell fate decisions, and its dysregulation has been 
associated with several diseases, such as cancer. However, its role in airway inflammation in asthma is less known. ${ }^{3}$ By analysing single nucleotide polymorphisms in different asthma clusters, Li et al . identified that the Notch4 receptor gene was significantly associated with lung function in asthma. ${ }^{4}$ Furthermore, Xia et al . explored cellular and molecular mechanisms of allergic airway inflammation. They found that the presence of pollutant ultrafine particles (UFPs) increased inflammation by promoting a Jag1-Notch4dependent interaction between alveolar macrophages (AMs) and allergen-specific CD4 T cells, thus increasing Th cell differentiation. ${ }^{5}$

Harb et al . focused on identifying Notch4-mediated immune tolerance disruption mechanisms that cause tissue inflammation in allergic asthma. ${ }^{2}$ They studied the impact of Notch4 dysregulation on immune tolerance in the context of allergic airway inflammation. First, they found that Notch4 is expressed on Treg cells in allergic airway inflammation. They discovered that the deletion of Notch4 in Treg cells in mice suppressed ovalbumin-specific IgE production along with lung tissue Th2 and Th17 cell responses. Further, the infiltration of $\mathrm{T}$ cells and eosinophils into the lung tissues was attenuated. They also observed that the Hippo and the Wnt pathway promoted Th17 and Th2 responses, respectively, in an in vitromodel. Additionally, to analyse the role of each of the Hippo and Wnt pathways in Treg cells, they performed a combined deletion of Yap1, Wntr1 (Hippo pathway) and Ctnnb1 (Wnt pathway) genes. In this case, they observed a suppression of Th2 and Th17 cell responses. Of note, this effect did not occur when the deletion was performed individually, demonstrating that the disruption of immune tolerance is dependent upon both pathways being affected concurrently. ${ }^{2}$ This may be construed as the reason why therapies targeting only Th17 pathway in asthma have not been clinically relevant. ${ }^{6}$

Notch4 expression also reduced the capacity of lung Treg cells to supress type 2 innate lymphoid cells (ILC2s) and activated the Wnt-cytokine growth and differentiation factor 15 (GDF15) axis, demonstrating an important role of the latter in promoting airway inflammation. These results support the critical role of Notch regulating ILC2 cell secretion of Th2 cytokines. Interestingly, Notch4 expression analysis in peripheral blood mononuclear cells of asthmatics revealed a differential increased expression in the Treg population, that was associated with clinical severity. ${ }^{2}$ The role of Tregs cells is perhaps more clinically relevant in immune tolerance and autoimmune responses in severe allergic asthma than in patients with mild allergic asthma. Indeed, dysregulation of Tregs in eosinophilic airways may underlie susceptibility and progression into autoinflammatory pathology, as observed in one-third of eosinophilic asthmatics. ${ }^{7}$ Moreover, autoantibodies against eosinophil cationic proteins, such as eosinophil peroxidase, can induce ILC2 to release Th2 cytokines via activation of death receptors. ${ }^{8}$ Taken together, the Notch4-GDF15 axis may be the transcriptional switch for this mechanism, warranting further investigation. Indeed, this signalling axis may be more clinically relevant in the severe patients in whom ILC2 cell biology may play a prominent role.

The authors also identified IL-6 as an inducer of Notch4 expression in the inducible Treg cells in the lung tissue, during allergen-specific differentiation (Figure 1 ). Interestingly, treatment with an anti-IL-6 receptor (IL-6R) monoclonal antibody suppressed Notch4 expression in a patient with severe asthma. This is consistent with anecdotal evidence from Etsy et al. where treatment with tocilizumab, a humanized anti-IL-6R monoclonal antibody, showed clinical improvement in two patients with severe asthma. ${ }^{9}$ The activation of cell types with no or low expression of membrane IL-6R depend on IL-6 trans-signalling. ${ }^{10}$ In this regard, increased IL-6R trans-signalling may increase airway eosinophilia by inducing IL-33 expression, which is a potent activator of mature eosinophils. ${ }^{10}$ These data suggest that IL- 6 targeting might be effective in selected patients with IL-4R polymorphism and a pleotropic airway cellular response.

With their findings, Harb et al . uncover the activation of the Notch4-Wnt-GDF15 axis in Treg cells, demonstrating that this axis acts by dysregulating its immunomodulatory function and promoting inflammation in allergic asthma. ${ }^{2}$ In brief, once the UFPs enter the airway, they are absorbed by the AMs causing IL-6 release, which ultimately promotes Notch4-receptor expression on Treg cells. Notch4 signalling activates the Hippo and Wnt pathways which can modify Treg cells leading into the activation of the Th2 and Th17 pathways. Furthermore, the Wnt pathway permits the release of GDF15 which promotes IL-13 production by ILC2, thus contributing to the inflammatory response (Figure 1 ). ${ }^{3}$ Harbet al . provide novel insights 
about the Notch4-Wnt-GDF15 axis in Treg cells functioning for the understanding of the pathogenesis of airway tissue inflammation, particularly the mechanisms involved in severe asthma. This may lead to new therapeutic strategies being explored that target Notch4 to restore immune tolerance in allergic asthma.

Conflict of interest : The authors declare that they have no conflicts of interest in relation to this manuscript.

Financial support : The authors received no specific funding for this work.

Authorship : All authors contributed to the manuscript, revised and edited.

References :

1. Amsen D, Helbig C, Backer RA. Notch in T Cell Differentiation: All Things Considered. Trends Immunol. 2015;36(12):802-814.

2. Harb H, Stephen-Victor E, Crestani E, et al. A regulatory T cell Notch4-GDF15 axis licenses tissue inflammation in asthma. Nat Immunol. 2020;21(11):1359-1370.

3. Hammad H, Lambrecht BN. Wnt and Hippo pathways in regulatory T cells: a NOTCH above in asthma. Nat Immunol. 2020;21(11):1313-1314.

4. Li X, Howard TD, Moore WC, et al. Importance of hedgehog interacting protein and other lung function genes in asthma. J Allergy Clin Immunol. 2011;127(6):1457-1465.

5. Xia M, Harb H, Saffari A, Sioutas C, Chatila TA. A Jagged 1-Notch 4 molecular switch mediates airway inflammation induced by ultrafine particles. J Allergy Clin Immunol. 2018;142(4):1243-1256 e1217.

6. Busse WW, Holgate S, Kerwin E, et al. Randomized, double-blind, placebo-controlled study of brodalumab, a human anti-IL-17 receptor monoclonal antibody, in moderate to severe asthma. Am J Respir Crit Care Med. 2013;188(11):1294-1302.

7. Mukherjee M, Bulir DC, Radford K, et al. Sputum autoantibodies in patients with severe eosinophilic asthma. J Allergy Clin Immunol.2018;141(4):1269-1279.

8. Machida K, Aw M, Salter BMA, et al. The Role of the TL1A/DR3 Axis in the Activation of Group 2 Innate Lymphoid Cells in Subjects with Eosinophilic Asthma. Am J Respir Crit Care Med.2020;202(8):1105-1114.

9. Esty B, Harb H, Bartnikas LM, et al. Treatment of severe persistent asthma with IL-6 receptor blockade. J Allergy Clin Immunol Pract.2019;7(5):1639-1642 e1634.

10. Jevnikar Z, Ostling J, Ax E, et al. Epithelial IL-6 trans-signaling defines a new asthma phenotype with increased airway inflammation.J Allergy Clin Immunol. 2019;143(2):577-590. 


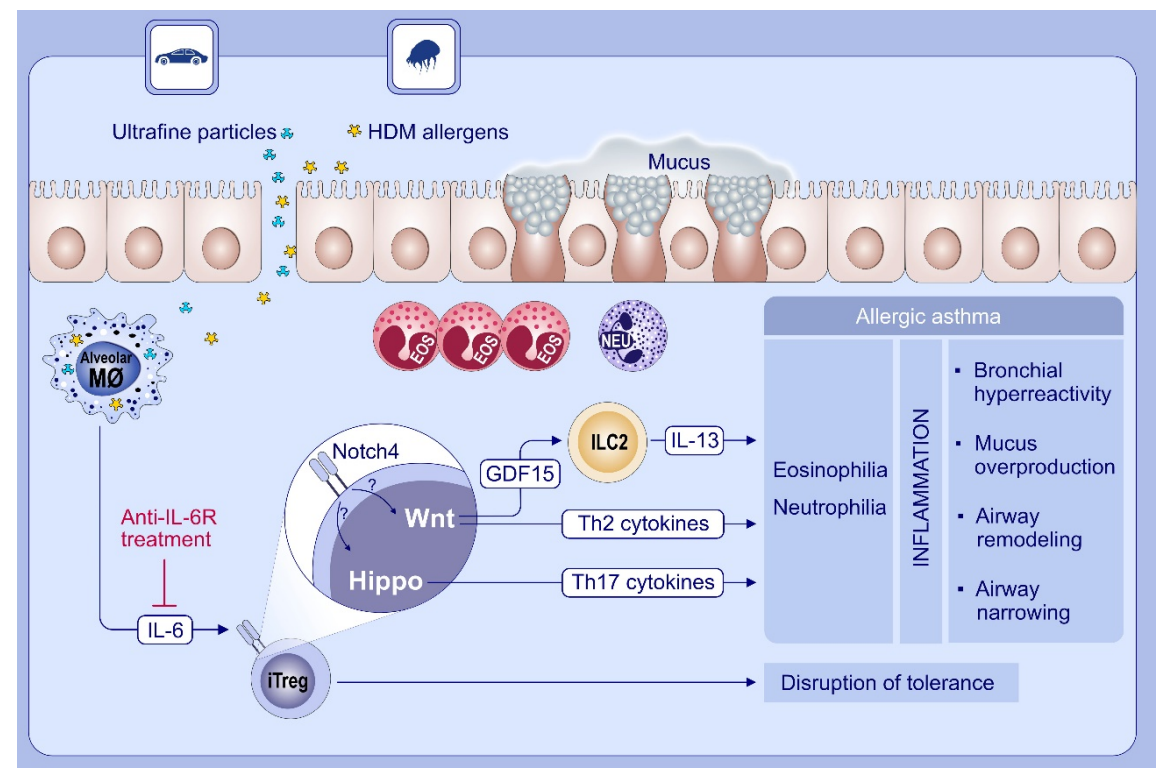

Figure 1. The Notch4-Wnt-GDF15 axis in Treg cells. The UFPs and HDM (house dust mite) allergens enter the airway and are removed by the AMs, which causes a release of IL-6 that promotes the expression of Notch4 receptor on Treg cells. Notch4 signalling activates the Hippo and Wnt pathways, which can modify Treg cells leading to the activation of the Th2 and Th17 pathways. This modification produces the dysregulation of the immune tolerance. The Wnt pathway may cause the release of GDF15, promoting IL-13 release by the ILC2, which in turn contributes to the inflammatory response in allergic asthma. 